Int. J. Dev. Biol. 50: 423-427 (2006)

doi: $10.1387 /$ ijdb.052097et

Short Communication

\title{
Identification of TH-B, a new oligomeric variant of the Xenopus morphogenetic factor, tumorhead
}

\author{
EDWIN E. TRAVERSO\#, MIN-SOON CHO\#, CHUAN-FEN WU and LAURENCE D. ETKIN* \\ Department of Molecular Genetics, University of Texas, M.D. Anderson Cancer Center, Houston, USA
}

\begin{abstract}
The Xenopus laevis gene tumorhead (TH) is a regulator of cell proliferation of the ectodermal germ layer during embryonic development. TH overexpression results in increased cell proliferation within the developing ectoderm, causing an expansion of the neural plate. Conversely, loss of TH function results in inhibition of proliferation of ectodermal cells. Embryos with altered levels of TH protein are unable to express neural differentiation markers, indicating that the effect of TH in proliferation is linked with differentiation in the nervous system. To date, the molecular mechanism by which TH affects cell proliferation during embryogenesis is unknown. We have utilized the yeast two-hybrid system to identify protein partners of TH that could lead us to define the mechanism or pathway through which TH functions. Using this assay we have identified a new variant of TH designated TH-B, as a potential protein partner of the original TH, now referred to as TH-A. The sequence for TH-B was found to be $85 \%$ identical at the amino acid level to the TH-A sequence. Further characterization of the TH-B variant using RT-PCR indicates that it is expressed ubiquitously throughout development from early cleavage stages until at least the tadpole stage. TH-B association with TH-A was confirmed in co-immnoprecipitation studies in Xenopus, indicating that the two variants may function as an oligomer in vivo. These studies reveal the presence of an isoform of TH that may possess novel functional capabilities.
\end{abstract}

KEY WORDS: Xenopus laevis, isoform, cell proliferation, tumorhead, TH-B

The development of germ layers into their tissue derivatives is a complex process involving determination signals that alter the proliferation status of the cells and induce them to follow a specific differentiation program. As the target cells receive these inductive signals, their cell cycle is downregulated, allowing them to differentiate. Therefore, proper coordination between cell proliferation and the processes of determination and differentiation must occur during embryogenesis. This coordination involves bi-directional mechanisms in which cell cycle components play different roles in the regulation of the processes of determination and differentiation and in other instances, factors for determination and differentiation regulate transitions that occur during the cell cycle (Ohnuma et al., 2001). In an analysis of maternally expressed genes that regulate early development in Xenopus laevis, Wu et al. (2001) identified a novel gene named tumorhead (TH). Further experiments indicated that this gene might be a key regulator and/or coordinator of the processes of cell proliferation and differentiation during the development of the ectodermal germ layer. Overexpression of $\mathrm{TH}$ results in expansion of the neural plate at the neurula stage and abnormal overgrowths in the head region at the tadpole stage. These alterations in the development of the nervous system are caused by hyperproliferation of ectodermal cells that are already committed to the neural pathway. In cells overexpressing $\mathrm{TH}$, expression of neural differentiation genes is not detected. However, expression of early markers of neural induction is not affected. Loss of $\mathrm{TH}$ function caused by the injection of morpholino antisense deoxyoligonucleotides into embryos, inhibits the proliferation of cells of ectodermal lineage, including the epidermis and the neural epithelium ( $\mathrm{Wu}$ et al., 2003). These cells do not express neural differentiation markers and as a consequence, a proper neural tube is not organized. The effects that $\mathrm{TH}$ overexpression and $\mathrm{TH}$ depletion have on neuroectoderm differentiation, although similar, might be mediated by different mechanisms. Loss of $\mathrm{TH}$ function may result in the lack of expression of differentiation markers through inhibition of cell division before the cells are competent to respond to differentiation signals. On the contrary, TH overexpression inhibits differentiation possibly by maintaining the cells in a continuous state of

Abbreviations used in this paper: $\mathrm{TH}$, tumorhead.

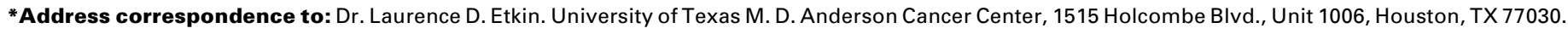
Fax: +1-713-834-6318. e-mail: Ide@mdanderson.org
}

\# Note: The indicated authors contributed equally to this work. 
proliferation, not allowing them to respond to differentiation factors. More recently, studies in which TH was expressed in the fission yeast Schizosaccharomyces pombe, demonstrated that $\mathrm{TH}$ affects the yeast cell growth rate, as well as modulates the cytoskeleton (Wu et al., 2004). Expression of TH in S. pombe results in severe morphological defects, including ovoid, bottleshaped and enlarged cells. TH expression causes cytoskeletal defects including depolarization of the cortical F-actin cytoskeleton and increased microtubule formation. The effects seen in yeast are similar to those observed in neural plate cells in Xenopus(Wu and Etkin, unpublished). These results suggest that in addition to regulating cell proliferation in Xenopus, TH may be a modulator of cytoskeletal dynamics during embryogenesis. We describe here the identification of a new variant of $\mathrm{TH}$, designated $\mathrm{TH}-\mathrm{B}$, which has the ability to oligomerize with the original version of the protein, now called $\mathrm{TH}-\mathrm{A}$, and that is expressed ubiquitously throughout embryogenesis. The sequence divergence between $\mathrm{TH}-\mathrm{A}$ and the newly uncovered $\mathrm{TH}-\mathrm{B}$ protein poses the possibility that these two isoforms may play slightly different roles during embryonic development and/or may be subjected to different protein regulatory mechanisms such as different posttranslational modifications or binding to different regulatory factors.

\section{Results and Discussion}

\section{Identification and cloning of $\mathrm{TH}-\mathrm{B}$}

The molecular mechanism by which $\mathrm{TH}$ affects cell proliferation and differentiation, and/or cytoskeletal dynamics during embryogenesis is unknown. To address this problem, we have utilized the yeast two-hybrid system (Chien et al., 1991) to identify protein partners of TH that can help us to define its mode of action. In our screen, we used the yeast strain HF7c, which contains the reporter genes HIS3 and lacZ to monitor protein/protein interactions. Yeast cells were transformed with the vector pGBT9-TH(87-487), which encodes a truncation mutant of TH that lacks the first 86 amino acids fused to the Gal4 DNA binding domain. The TH N-terminal deletion eliminates its acidic domain, which lies within residues 48-86 and confers $\mathrm{TH}$ the ability of activating reporter genes in the two-hybrid system without the need of the Gal4 transcriptional activation domain (data not shown). Cells carrying the $\mathrm{TH}$ expression plasmid were subsequently transformed with an egg cDNA library cloned into the pGAD10 vector, which produces the protein encoded by the cDNA insert fused to the Gal4 transcriptional activation domain. $7 \times 10^{6}$ transformants were screened for HIS3 reporter gene activation and positive colonies were subsequently tested for lac $Z$ activation using filter-lift $\beta$-galactosidase assays. Plasmid DNA was isolated from positive clones and retransformed into SFY526 yeast cells to corroborate the two-hybrid interaction with $\mathrm{TH}$. Positive clones were sequenced to reveal the identity of the plasmid insert. Among the proteins that were identified in our screen is $\mathrm{TH}$ itself. TH

\section{TH-A}

$(87-487) /$

AD alone

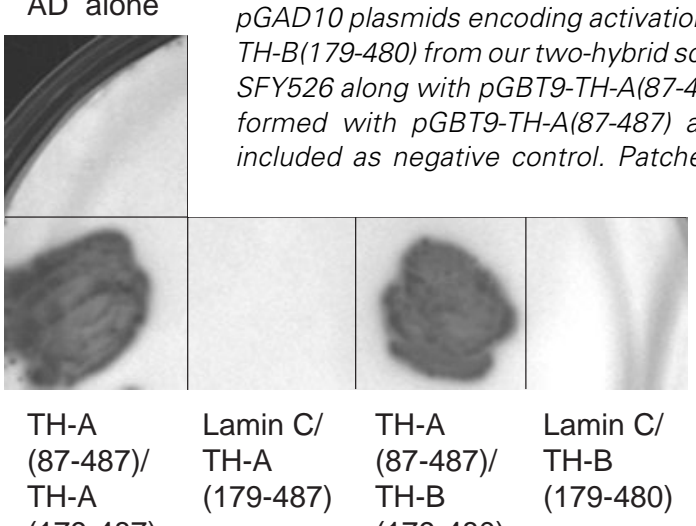

$(179-487)$ has the ability to oligomerize in the two-hybrid system (Wu and Etkin, unpublished observations), suggesting that it functions as a multimer in vivo. In addition, using the two-hybrid system we found a new variant of $\mathrm{TH}$, called $\mathrm{TH}-\mathrm{B}$, that is able to oligomerize with the original $\mathrm{TH}$, now designated as $\mathrm{TH}-\mathrm{A}$. Fig. 1 shows the results from filter-lift $\beta$-galactosidase assays to detect the activation of lacZexpression in SFY526 yeast cells expressing $\mathrm{TH}-\mathrm{A}$ and $\mathrm{TH}-\mathrm{B}$ fusion proteins. Cells containing TH-A DNA binding domain fusion protein in the presence of activation domain alone were included as negative control. We also used a plasmid for the expression of Lamin $C$ fused to the DNA binding domain as a control to discriminate against nonspecific reporter gene activation by $\mathrm{TH}-\mathrm{A}$ or $\mathrm{TH}-\mathrm{B}$ activation domain fusion proteins. In these experiments, we detected /ac $Z$ expression activation when $\mathrm{TH}-\mathrm{A}(179-487)$ or $\mathrm{TH}-\mathrm{B}(179-480)$ fused to the activation domain was present in cells expressing TH-A(87-487), but not Lamin C, fused to the DNA binding domain. This indicated a $\mathrm{TH}-\mathrm{A}$-dependent activation of the lac $Z$ reporter gene for both $\mathrm{TH}-\mathrm{A}$ and $\mathrm{TH}-\mathrm{B}$ activation domain fusion proteins.

The $\mathrm{TH}-\mathrm{B}$ clone isolated in the two-hybrid screen encodes residues 179-480. Therefore, we used the technique of 5' Rapid Amplification of cDNA Ends (5' RACE) to obtain the 5' end of the $\mathrm{TH}-\mathrm{B}$ message. Using primers designed according to the sequence of the TH-B clone obtained in our screen, we were able to obtain 5' RACE products that extended $\sim 300$ residues upstream from the starting codon (data not shown). This allowed us to assemble the entire mRNA sequence for TH-B (GenBank accession number DQ172837). The sequence for TH-B was found to be $89 \%$ identical at the nucleotide level and $85 \%$ identical at the amino acid level with the TH-A sequence. Fig. 2 shows an alignment of the amino acid sequences of $\mathrm{TH}-\mathrm{A}$ and TH-B. Several motifs had been defined previously for the TH-A protein (Wu et al., 2001). These include an acidic domain located within residues 48-86, a basic domain located within residues 165-178, a second basic domain located within the six C-terminal residues of the polypeptide (amino acids 482-487) and a predicted coiled coil domain redefined to be located within residues 284-314 using the COILS program (Lupas, 1996). The amino acid sequence alignment of the TH proteins shows a general conservation of all the $\mathrm{TH}-\mathrm{A}$ domains in the 


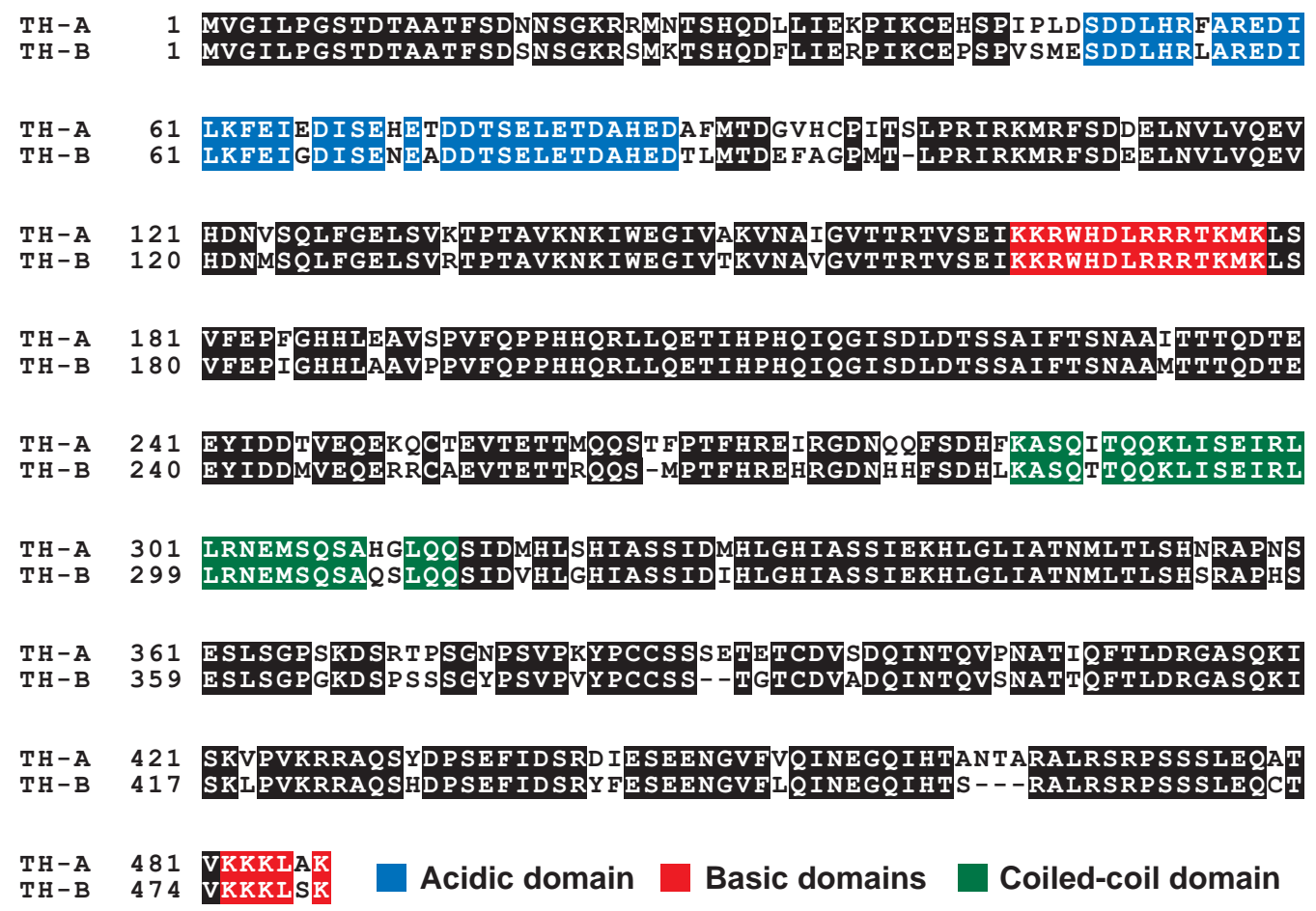

Fig. 2. Sequence alignment of TH variants. Amino acid sequence alignment of the TH-A and TH-B variants performed using the ClustalW program. Identical amino acids are boxed. The location of the different domains within TH-A and TH-B sequences is indicated as follows (amino acid positions given for TH-A sequence): N-terminal acidic domain (amino acids 48-86), blue; basic domains (amino acids 165-178 and 482-487), red; predicted coiledcoil domain (amino acids 284-314), green.

TH-B protein sequence, although the TH-B sequence has a more robust prediction for the formation of a coiled-coil domain than the $\mathrm{TH}-\mathrm{A}$ sequence.

\section{Temporal and spatial expression of TH variants}

Next, we analyzed the expression of both $\mathrm{TH}$ variants during Xenopus embryogenesis. Expression of $\mathrm{TH}-\mathrm{A}$ and $\mathrm{TH}-\mathrm{B}$ was detected by RT-PCR using total RNA preparations from embryos at various developmental stages and specific primers for the different isoforms (Fig. 3). We also used primers for the amplification of $\mathrm{eF} 1 \alpha$ as an internal control in our experiments. The lack of PCR products in reactions performed in the absence of reverse transcriptase ruled out the presence of contaminant DNA in our preparations. As expected, we observed that eF1 $\alpha$ was expressed throughout embryogenesis with much higher expression levels at stages after the mid blastula transition (stages 11-40) (Krieg et al., 1989). With respect to TH expression, we observed that both variants of $\mathrm{TH}$ are expressed during embryogenesis from the unfertilized egg until at least the tadpole stage, although exhibiting different levels of expression. While TH-B expression levels appear to remain constant from the egg until the tadpole stage (stage 40), TH-A expression levels are higher from the egg until the gastrula stage (stage 11), at which point its expression decreases to a level comparable to that of TH-B.

To analyze the spatial distribution of the different $\mathrm{TH}$ variants, embryos at various developmental stages were collected and dissected into their animal and vegetal halves, or dorsal and ventral regions. Expression of the $\mathrm{TH}-\mathrm{A}$ and $\mathrm{TH}-\mathrm{B}$ variants was detected by RT-PCR using the different RNA preparations as templates and the variant specific primer sets mentioned above (Fig. 4). PCR reactions for the amplification of TH-B were also performed in the absence of reverse transcriptase to assess the presence of contaminant DNA in our preparations. In these analyses, we observed that both $\mathrm{TH}-\mathrm{A}$ and $\mathrm{TH}-\mathrm{B}$ variants were expressed ubiquitously throughout the embryo at developmental stages ranging from the early blastula until the tailbud. Previous data indicated that the $\mathrm{TH}$ protein was enriched in the neural plate (Wu et al., 2001). These studies along with the temporal analysis of $\mathrm{TH}-\mathrm{A}$ and $\mathrm{TH}-\mathrm{B}$ expression presented here, suggest that the translation of the TH-A and TH-B mRNAs might be repressed in mesodermal and endodermal derivatives during neurulation.

\section{TH-A interacts with TH-B in Xenopus embryos}

To confirm the two-hybrid interaction between TH-A and TH-B in Xenopus embryos we employed coimmunoprecipitation assays. For these experiments, the expression vectors pCS2+MT$\mathrm{TH}-\mathrm{A}$ and $\mathrm{pCS} 2+\mathrm{NHA}-\mathrm{TH}-\mathrm{B}$ were created to synthesize transcripts encoding epitope-tagged versions of both $\mathrm{TH}$ variants. We injected embryos with myc-TH-A mRNA in the presence or absence of HA-TH-B mRNA. Fig. 5A shows that myc-TH-A was retained only in the anti-HA beads incubated with cell extracts containing HA-TH-B and not in the beads in which HA-TH-B was absent. In a coimmunoprecipitation experiment performed in the opposite configuration, we injected embryos with HA-TH-B mRNA in the presence or absence of myc-TH-A mRNA. Fig. 5B shows that $\mathrm{HA}-\mathrm{TH}-\mathrm{B}$ was retained in the anti-myc beads containing myc$\mathrm{TH}-\mathrm{A}$, but in was absent in the beads that did not contain myc- $\mathrm{TH}-$ A. These results confirm the interaction between $\mathrm{TH}-\mathrm{A}$ and $\mathrm{TH}-\mathrm{B}$ 

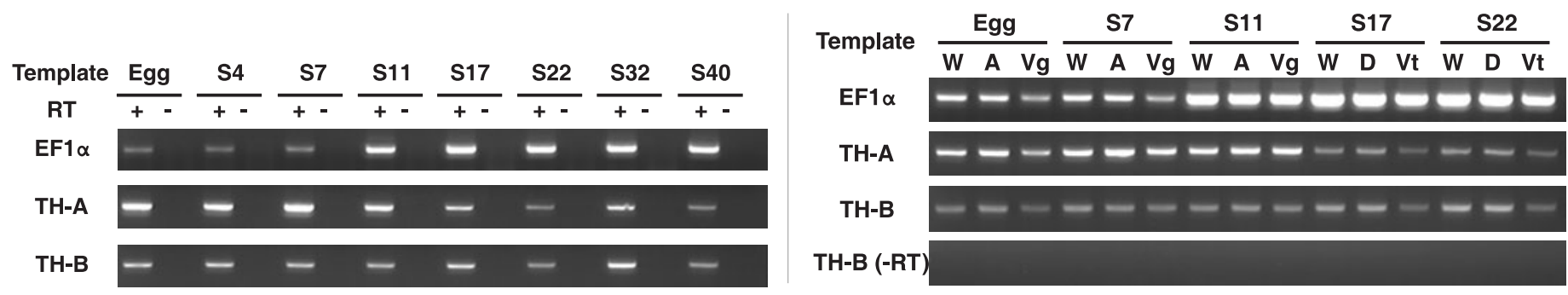

Fig. 3 (Left column). Temporal analysis of TH variants expression during embryogenesis. Embryos at the indicated developmental stages were collected and total RNA preparations were made from cell extracts from these embryos. Expression of the TH-A and TH-B variants was detected by $R T$-PCR using the different RNA preparations as templates and specific primer sets for the different isoforms. 1.2 $\mu$ g of total RNA was used as template for each reaction. PCR with primers for the amplification of the eF1 $\alpha$ message was performed as an internal experimental control. Reactions were performed in the presence (+) or absence (-) of reverse transcriptase to assess the presence of contaminant DNA in our preparations. Amplified PCR products were analyzed in a $2 \%$ agarose gel.

Fig. 4 (Right column). Spatial analysis of expression of TH variants during embryogenesis. Embryos at the indicated developmental stages were collected and dissected into their animal (A) and vegetal (Vg) hemispheres, or dorsal (D) and ventral (Vt) regions, according to the developmental stage. Total RNA preparations were made from cell extracts from whole (W) embryos or the embryos that were dissected. Expression of the TH-A and TH$B$ variants was detected by RT-PCR using the RNA preparations and specific primer sets for the different isoforms. 800 ng of total RNA was used as template for each reaction. PCR reactions for the amplification of TH-B were also performed in the absence of reverse transcriptase (-RT) to determine if contaminant DNA was present in our preparations. Reactions containing primers for the amplification of the eF1 $\alpha$ message were included as an internal control. Amplified PCR products were analyzed in a $2 \%$ agarose gel.

observed in the two-hybrid system and suggest that these $\mathrm{TH}$ isoforms may function as an oligomer during embryogenesis.

The studies presented herein describe the existence of a novel isoform of $\mathrm{TH}, \mathrm{TH}-\mathrm{B}$ and show that this $\mathrm{TH}$ variant, similarly to $\mathrm{TH}$ $\mathrm{A}$, is expressed ubiquitously in Xenopus embryos throughout embryogenesis. TH-B binds to TH-A both in yeast two-hybrid assays and in Xenopus embryos suggesting that the TH proteins function as oligomeric proteins. The presence of two different isoforms for TH in $X$. laevis might be due to the pseudotetraploid character of this organism (Kobel and Du Pasquier, 1986). In $X$. laevis, two different nonallelic paralogs have been described for most genes, which arose during evolution as a result of an ancient genome duplication event (Bisbee et al., 1977). The presence of two paralogs sometimes can lead to pseudogene formation, in which one of the duplicated genes is inactivated. However, different paralogs in Xenopusare more likely to remain active and eventually develop new gene functions rather than taking the pseudogene fate (Hughes and Hughes, 1993).

TH-A has been shown previously to be a positive regulator of cell proliferation and a modulator of the cytoskeleton during Xenopus development. It will be of great importance to determine if the newly identified TH-B variant regulates these aspects of cell

Fig. 5. TH-B interacts with TH-A in Xenopus embryos. Embryos at the two-cell stage were injected with myc-TH-A mRNA, HA-TH-B mRNA, or a combination of both transcripts. Embryos were grown to the neurula stage (stage 17) and cell extracts were made. Fractions of the lysates were removed to analyze the expression of the myc-TH-A and HA-TH-B proteins. The lysates were then incubated with anti-HA antibody (A), or anti-myc antibody (B), followed by the addition of protein-A and protein$G$ sepharose beads to trap the immune complexes. After washing the beads, these were boiled in SDS-PAGE loading buffer. The lysate (L) and the immunoprecipitate (IP) samples were run in 10\% SDS polyacrylamide gels, followed by anti-HA or anti-myc Western blots. Note the presence of myc-TH-A only in the HA-IP containing HA-TH-B $(A)$ and the presence of $H A-T H-B$ only in the myc-IP containing myc-TH-A (B). function like TH-A does, or if it plays roles in other cellular functions. An interesting possibility is that both TH variants can be subjected to different modes of protein regulation. The TH variants may bind to different regulatory factors, or may be regulated by different posttranslational modifications. For instance, several predicted phosphorylation and sumoylation sites in TH-A are not conserved in TH-B, whereas several predicted TH-B phosphorylation sites are not present in TH-A. Different regulatory capabilities between the TH proteins would enhance their potential in executing different roles during embryogenesis. In addition, it will be of interest to determine if $\mathrm{TH}-\mathrm{A} / \mathrm{TH}-\mathrm{B}$ oligomerization is necessary for $\mathrm{TH}$ function during development. A possible scenario is that different combinations of TH multimers may function differentially. Further characterization of both $\mathrm{TH}$ variants will likely expand our knowledge about the relationship between the regulation of cell proliferation and the control of cell differentiation during embryonic development.

A

\begin{tabular}{lclll} 
& \multicolumn{3}{c}{ HA IP } \\
\cline { 2 - 5 } myc-TH-A mRNA & + & + & + & + \\
HA-TH-B mRNA & - & - & + & + \\
& & IP & L & IP \\
myc blot & & & &
\end{tabular}

B

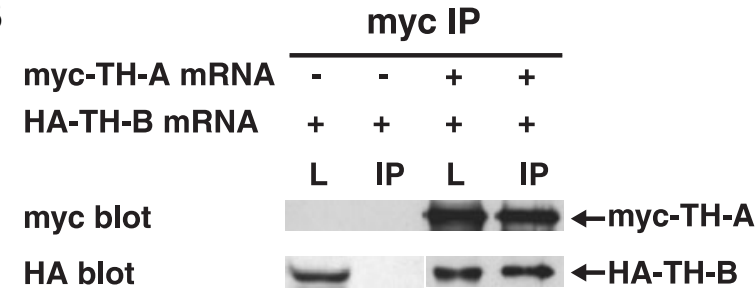




\section{Experimental Procedures}

\section{Construction of plasmids}

To create pGBT9-TH(87-487), codons 87-487 of TH-A were amplified by PCR using primers with linkers that facilitated their insertion into pGBT9 (BD Biosciences) following standard cloning techniques. To create pCS2+MT-TH-A the ORF of TH-A was amplified by PCR using primers with linkers for ligation into the EcoRI/Xhol sites of pCS2+MT (kindly provided by Dave Turner, University of Michigan). To obtain pCS2+NHA-TH-B, codons $179-480$ of TH-B were amplified by PCR using as template the pGAD10-TH-B(179-480) plasmid isolated from our twohybrid screen. The amplified PCR product was then ligated into the EcoRI/Xhol sites of pCS2+NHA (kindly provided by Pierre D. McCrea, UT MD Anderson Cancer Center) to create pCS2+NHA-TH-B(179-480). After obtaining the 5' end of the TH-B message through 5' Race (see below), codons 1-311 were amplified by PCR and ligated into the EcoRI/ Pstl sites of pCS2+NHA-TH-B(179-480) to create pCS2+NHA-TH-B. Transcripts were synthesized in vitro from these plasmids using the mMessage mMachine reagents (Ambion).

\section{Yeast two-hybrid screen and assays}

The Matchmaker two-hybrid system (BD Biosciences) was used to identify TH-A binding proteins. Yeast strain HF7c was sequentially transformed with pGBT9-TH(87-487) and an egg cDNA library cloned into pGAD10 (BD Biosciences) following the high efficiency yeast transformation protocol described by Gietz and Woods (2002). After transformation with the cDNA library, the cells were plated in media lacking Leu and Trp to select for transformants and His to assess HIS3 reporter gene activation. Plates contained $5 \mathrm{mM}$ 3-aminotriazole to suppress background growth that results from some basal expression of the HIS3 gene. Colonies were allowed to grow for 8 days at $30^{\circ} \mathrm{C}$. Positive colonies obtained in the screen were assayed in filter lift $\beta$-galactosidase assays for lacZ activation (Ausubel et al., 1997). Patches from these colonies were transferred to nylon membranes, which were treated with liquid nitrogen to permeabilize the cells. The filters were incubated at $30^{\circ} \mathrm{C}$ in $\mathrm{Z}$ buffer $\left(60 \mathrm{mM} \mathrm{Na}_{2} \mathrm{HPO}_{4}, 40 \mathrm{mM} \mathrm{NaH}_{2} \mathrm{PO}_{4}, 10 \mathrm{mM} \mathrm{KCl}, 1 \mathrm{mM} \mathrm{MgSO}{ }_{4}, 50\right.$ $\mathrm{mM} 2$-mercaptoethanol) containing 5-bromo-4-chloro-3-indolyl $\beta$-Dgalactopyranoside (X-GAL) as substrate.

\section{5'RACE}

To obtain the 5' end of the TH-B message, we used the 5' RACE System for Rapid Amplification of cDNA Ends (Invitrogen) following the protocol suggested by the manufacturer.

\section{Expression analysis by $R T-P C R$}

Total RNA was extracted from whole or dissected embryos using the Trizol reagent (Invitrogen). Embryos were homogenized in Trizol using a ratio of $100 \mu \mathrm{l}$ of reagent per embryo. Extracted RNA was treated with TURBO DNA-free (Ambion) to eliminate contamination with genomic DNA. RT-PCR was performed using the SuperScript III One-Step RTPCR System with Platinum TaqDNA Polymerase reagents from Invitrogen. First strand $\mathrm{CDNA}$ synthesis was performed at $55^{\circ} \mathrm{C}$ for $30 \mathrm{~min}$ followed by 25 cycles of PCR. Amplification of TH-A and TH-B was carried out using the sense primer CTG TTA CAG GAG ACT ATA CAT CCC C for both variants and the antisense primers TCC CAG AGG GGG TAC GGG AGT CTT TAC T for TH-A and ATC CAG AAG AGC TAG GGG AGT CTT TAC $C$ for TH-B. This yielded PCR products of $514 \mathrm{bp}$ and $520 \mathrm{bp}$ for TH-A and TH-B, respectively.

\section{Coimmunoprecipitation studies}

Embryos at the two-cell stage were injected with $3 \mathrm{ng}$ of myc-TH-A mRNA, $3 \mathrm{ng}$ of HA-TH-B mRNA, or a combination of both transcripts. Embryos were grown to the neurula stage (stage 17) and cell extracts were made from 15 embryos in $150 \mu$ l of extraction buffer $(10 \mathrm{mM}$ Hepes, $\mathrm{pH} 7.4,150 \mathrm{mM} \mathrm{NaCl}, 2 \mathrm{mM}$ EDTA and $2 \mathrm{mM}$ EGTA). $50 \mu$ fractions of the lysates were removed, mixed with $50 \mu \mathrm{l}$ of $2 X$ SDS-PAGE loading buffer and boiled for $5 \mathrm{~min}$. This allowed us to analyze the expression of the myc-TH-A and HA-TH-B proteins by SDS-PAGE and Western blotting. Extraction buffer was added to the remaining portion of the lysates to a final volume of $890 \mu \mathrm{l}$. Samples were then incubated with $10 \mu \mathrm{l}$ of antiHA antibody (Santa Cruz) or anti-myc antibody (Santa Cruz) for $1 \mathrm{hr}$, followed by the addition of $100 \mu \mathrm{l}$ of a $50 \%$ slurry mixture (1:1) of protein$A$ and protein-G sepharose beads. Samples were mixed by inversion for $1 \mathrm{hr}$. After washing the beads four times in extraction buffer containing $0.5 \% \mathrm{TX}-100$, these were boiled in $50 \mu \mathrm{l}$ of 2 X SDS-PAGE loading buffer for $5 \mathrm{~min}$. Aliquots $(20 \mu \mathrm{l})$ of the lysate and the immunoprecipitate samples were run in $10 \%$ SDS polyacrylamide gels, followed by immunoblotting with anti-HA or anti-myc antibodies.

\section{Acknowledgements}

We thank Dr. Malgosia Kloc for technical advice and critical comments on the manuscript. We would also like to acknowledge support from the core grant (CA 16672) DNA sequencing facility. This work was partially supported by grants from the March of Dimes (FY99-0347-3) and NSF (IBN 0318768-02) to LDE and the NIH (5 T32 HD007325 18; 5 F32 GM71103) to EET.

\section{References}

AUSUBEL, F.M., BRENT, R., KINGSTON, R.E., MOORE, D.D., SEIDMAN, J.G., SMITH, J.A., STRUHL, K. (1997). Current Protocols in Molecular Biology, John Wiley \& Sons, New York.

BISBEE, C.A., BAKER, M.A., WILSON, A.C., HAJI-AZIMI, I., FISCHBERG, M (1977). Albumin phylogeny for clawed frogs (Xenopus). Science 195: 785-787.

CHIEN, C.T., BARTEL, P.L., STERNGLANZ, R., FIELDS, S. (1991). The two-hybrid system: a method to identify and clone genes for proteins that interact with a protein of interest. Proc. Natl. Acad. Sci. USA 88: 9578-9582.

GIETZ, R.D., WOODS, R.A. (2002). Transformation of yeast by lithium acetate/ single-stranded carrier DNA/polyethylene glycol method. Methods Enzymol. 350: 87-96.

HUGHES, M.K., HUGHES, A.L. (1993). Evolution of duplicate genes in a tetraploid animal, Xenopus laevis. Mol. Biol. Evol. 10: 1360-1369.

KOBEL, H.R, DU PASQUIER L. (1986). Genetics of polyploidy Xenopus. Trends Genet. 2: 310-315.

KRIEG, P.A., VARNUM, S.M., WORMINGTON, W.M., MELTON, D.A. (1989). The mRNA encoding elongation factor 1 -alpha (EF-1 alpha) is a major transcript at the midblastula transition in Xenopus. Dev. Biol. 133: 93-100.

LUPAS, A. (1996). Prediction and analysis of coiled-coil structures. Methods Enzymol. 266: 513-525.

OHNUMA, S-I., PHILPOTT, A., HARRIS, W. (2001). Cell cycle and cell fate in the nervous system. Curr. Opin. Neurobiol. 11: 66-73.

WU, C.F., CHAN, A., ETKIN, L.D. (2003). Difference in the maternal and zygotic contributions of tumorhead on embryogenesis. Dev. Biol. 255: 290-302.

WU, C.F., NAKAMURA, H., CHAN, A.P-Y., ZHOU, Y-H., CAO, T., KUANG, J., GONG, S-G., HE, G., ETKIN, L.D. (2001). Tumorhead, a maternal gene product that effects neural differentiation through regulation of proliferation. Development 128: 3381-3393.

WU, C.F., YANG, P., TRAVERSO, E.E., ETKIN, L.D., MARCUS, S. (2004). The Xenopus laevis morphogenetic factor, tumorhead, causes defects in polarized growth and cytokinesis in the fission yeast, Schizosaccharomyces pombe. Biochem. Biophys. Res. Commun. 325: 439-444. 